\title{
Management of 21 hydroxylase deficiency salt-wasting form of congenital adrenal hyperplasia
}

\author{
K S H de Silva ${ }^{1}$ \\ Sri Lanka Journal of Diabetes Endocrinology and Metabolism 2011; 1: 28-29
}

\section{Introduction}

Congenital adrenal hyperplasia (CAH) is a group of autosomal recessively inherited disorders of impaired adrenal steroid synthesis. Approximately $95 \%$ of CAH is due to deficiency of 21-hydroxylase enzyme resulting from mutations in the CYP21 A2 gene and is known as classical CAH (1) with a world-wide incidence of 1:10,000 to 1:20,000 births. Aldosterone deficiency in addition to deficiency of cortisol and excess androgens is seen in about $75 \%$ of classical CAH and results in the salt-wasting form of 21 hydroxylase deficiency $\operatorname{CAH}(2,3)$.

Cortisol deficiency with excess production of androgens is seen in the simple virilizing form of 21 hydroxylase deficiency CAH. $3 \beta$ hydroxysteroid dehydrogenase ( $3 \beta \mathrm{HSD}$ ) deficiency will result in varying degrees of deficiency of cortisol and aldosterone and cause ambiguous genitalia in a baby girl due to accumulation of DHEAS and inadequate virilization in a baby boy due to lack of testosterone.

\section{Presentation of the salt-wasting form of CAH}

A baby girl with this condition would be virilized with ambiguous genitalia (Table 1) and thus it would be difficult to identify the sex of the baby at birth. There will be progressive pigmentation and evidence of salt loss would manifest after the 1st week of life $(4,5)$. There will be a significant weight loss of $>10 \%$ of the birth weight with poor feeding, vomiting and dehydration with characteristic biochemical abnormalities. Such a baby would collapse and die unless appropriate treatment is commenced.

In an affected baby boy the diagnosis would be missed at birth as genital ambiguity is not a feature and therefore be made only subsequently when presenting with features of adrenal cortical insufficiency.

Table 1. Appearance of the external genitalia in a baby girl with congenital adrenal hyperplasia
Clitoromegaly
Pigmented, rugose, 'labioscrotal' folds
Posterior labial fusion or total fusion
One / two perineal openings
No palpable gonads
Normal anus

The initial investigations that are indicated and the expected results are summarized in Table 2. Neonatal sepsis being an important differential diagnosis, a blood culture and CRP are indicated.

A buccal smear for Barr bodies is a useful preliminary investigation as the result is available within 24 hours and a tentative answer could be given to the parents' anxious query regarding the possible sex of their baby. Nevertheless a karyotype is mandatory to confirm genetic sex.

Table 2. Investigations in salt-wasting congenital adrenal hyperplasia

\begin{tabular}{|c|c|}
\hline \multirow[t]{3}{*}{ Biochemistry } & $\begin{array}{l}\text { Serum electrolytes - hyponatraemia } \\
\text { and hyperkalaemia }\end{array}$ \\
\hline & Blood glucose - low \\
\hline & $\begin{array}{l}17 \text { hydroxyprogesterone }(17 \mathrm{OHP})^{*} \\
\text { - very high }\end{array}$ \\
\hline $\begin{array}{l}(* \text { preferably } \\
\text { taken at } 0800 \mathrm{hrs})\end{array}$ & $\begin{array}{l}\text { DHEAS* and Testosterone* } \\
\text { higher than expected for sex and age }\end{array}$ \\
\hline $\begin{array}{l}\text { Ultrasonography } \\
\text { (in a girl) }\end{array}$ & $\begin{array}{l}\text { Bilateral adrenal hyperplasia, uterus } \\
\text { and ovaries present, kidneys normal }\end{array}$ \\
\hline
\end{tabular}

\section{Initial management}

Definitive management is with intravenous normal saline and hydrocortisone during the initial 24-48 hours followed by oral hydrocortisone and fludrocortisone with or without oral salt supplementation.

The 17 OHP should be repeated in 2 weeks and thereafter the doses of hydrocortisone and fludrocortisone are calculated for the body surface area. Hydrocortisone is ideally given 3 times a day and fludrocortisone either once or twice a day.

\section{Long term management}

Follow up is based on clinical features, anthropometry, biochemistry and radiology. Affected girls will also require surgical intervention.

${ }^{1}$ Senior Lecturer in Paediatrics, Faculty of Medicine, Colombo, Sri Lanka. 


\section{Clinical features and anthropometry}

Meticulous monitoring of length / height, weight and OFC is an essential aspect of follow up. Increase in pigmentation and deviation of the growth centile (length) with an increase in 17 OHP indicate inadequate hydrocortisone replacement whereas cushingoid facies with a suboptimal growth velocity would indicate over treatment $(1,6)$.

In the absence of easy access to plasma renin activity (PRA), salt preference/craving, inadequate weight gain with electrolyte abnormalities and changes in the blood pressure, are helpful to assess the adequacy of treatment with fludrocortisone (6).

\section{Biochemistry}

Regular estimations of $17 \mathrm{OHP}$ and serum electrolytes should be done and are used together with the clinical features and blood pressure to adjust the drug doses.

\section{Radiology}

Bone age estimations are useful to monitor skeletal maturation which should ideally be compatible with the chronological age ensuring attainment of a height within the target range for the child. A delayed or advanced bone age would indicate over or under treatment respectively with hydrocortisone when an advanced bone age will compromise the final adult height achieved.

\section{Surgery}

Baby girls will need endoscopic evaluations and contrast studies in late infancy followed by feminizing genitoplasty with subsequent vaginal dilatations when necessary $(1,5,7)$.

\section{Counseling}

The process of counseling can be initiated once the clinical diagnosis is made and the possible sex of the baby is known by the presence of Barr bodies in the buccal smear. Once the condition is confirmed and the karyotype is available, a detailed discussion with the parents regarding all aspects of the disease must be undertaken. The importance of clinic attendance and compliance with the medication that has to be taken life long, the necessity to perform regular blood investigations, 'sick day' management and the need for surgery should all be discussed at length with the parents and reinforced periodically during clinic visits.

Psychological support for the parents and child is essential and should be made available on a regular basis.

\section{Treatment during adolescence}

Once the child attains puberty and growth is completed, the hydrocortisone can be changed to a daily dose of dexamethasone at night. Most patients can manage without fludrocortisone by modifying the dietary intake of salt.

\section{Prenatal treatment of $\mathrm{CAH}$}

Virilization of the genitalia of an affected female fetus begins approximately 6 weeks after conception. Therefore if prenatal treatment is considered, dexamethasone should be started as soon as possible and will only be beneficial and minimize virilization if the fetus is an affected female(1).

\section{Future pregnancies}

21 hydroxylase deficiency $\mathrm{CAH}$ is inherited as an autosomal recessive disorder. Therefore the parents should be counselled that for each pregnancy there is a $25 \%$ chance of the baby being affected. They should also be informed that antenatal diagnosis for this condition is not possible in this country and that abortion is not legal.

\section{References}

1. Spieser PW, Azziz R, Baskin LS, Ghizzoni L, et al. Congenital adrenal hyperplasia due to steroid 21-hydroxylase deficiency. An Endocrine Society Clinical Practice Guideline. J Clin Endocrinol Metab 2010; 95(9): 4133-60.

2. Brown J, Warne G. Practical management of the intersex infant. J Pediatr Endocrinol Metab 2005; 18(1): 3-23.

3. Huma Z, Crawford C, New MI. Congenital adrenal hyperplasia. In: Brook CGD editor. Clinical Paediatric Endocrinology. Blackwell Science Ltd, 1995: 536-57.

4. Warne GL, Hughes IA. The clinical management of ambiguous genitalia. In: Brook CGD editor. Clinical Paediatric Endocrinology. Blackwell Science Ltd, 1995: 53-68.

5. Warne GL. Evaluation of a child with ambiguous genitalia diagnosis and management. In: Desai Meena, Menon PSN, Bhatia V editors. Pediatric Endocrine Disorders. Chennai: Orient Longman Private Ltd, 2008: 401-24.

6. Dauber A, Kellogg M, Majzoub JA. Monitoring of therapy in congenital adrenal hyperplasia. Clinical Chemistry 2010; 56: $8,1245-51$.

7. Warne G, Grover S, Hutson J, et al. Murdoch Children's Research Institute Sex Study Group. A long term outcome study of intersex conditions. J Pediatr Endocrinol Metab 2005; 18(6): 555-67. 